
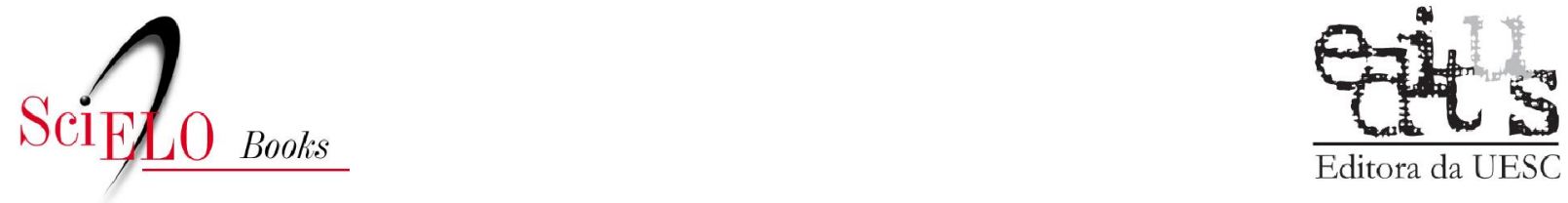

Editora da UESC

\title{
24 - Interações entre Poneromorfas e fontes de açúcar na vegetação
}

\author{
Thamy Evellini Dias Marques \\ Gabriela Castaño-Meneses \\ Cléa dos Santos Ferreira Mariano \\ Jacques H. C. Delabie
}

SciELO Books / SciELO Livros / SciELO Libros

MARQUES, TED., et al. Interações entre Poneromorfas e fontes de açúcar na vegetação. In:

DELABIE, JHC., et al., orgs. As formigas poneromorfas do Brasil [online]. Ilhéus, BA: Editus, 2015,

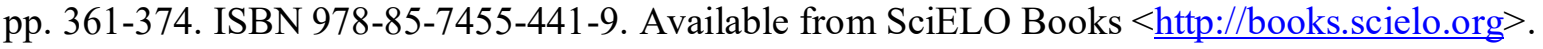

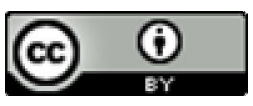

All the contents of this work, except where otherwise noted, is licensed under a Creative Commons Attribution 4.0 International license.

Todo o conteúdo deste trabalho, exceto quando houver ressalva, é publicado sob a licença Creative Commons Atribição 4.0.

Todo el contenido de esta obra, excepto donde se indique lo contrario, está bajo licencia de la licencia Creative Commons Reconocimento 4.0. 


\title{
Interações entre Poneromorfas e fontes de açúcar na vegetação
}

\author{
Thamy Evellini Dias Marques, Gabriela Castaño-Meneses, \\ Cléa dos Santos Ferreira Mariano, Jacques H. C. Delabie
}

\section{Resumo}

Mutualismo é uma importante interação ecológica em que ambas as espécies são beneficiadas pela associação. Tal associação pode ser obrigatória ou facultativa, sendo obrigatória quando a sobrevivência de pelo menos uma das espécies envolvidas depende da associação. Associações mutualísticas podem ser classificadas ainda como diretas e indiretas. São consideradas como associações mutualísticas diretas quando as espécies envolvidas na interação estão diretamente em contato físico; já nas associações mutualísticas indiretas, as espécies envolvidas na associação se beneficiam sem interagir fisicamente. Associações mutualísticas são extremamente comuns entre formigas e plantas, uma vez que tais grupos são abundantes e altamente diversos ao redor do mundo. Em geral, nessas interações, as formigas encontram recursos nas plantas como locais de nidificação e alimento (e.g. líquidos açucarados), e, em contrapartida, as formigas defendem a planta contra herbívoros. Dessa maneira, as interações entre formigas e nectários extraflorais das plantas exemplificam associações mutualísticas diretas. Já as interações entre formiga-hemíptero-planta podem exemplificar interações mutualísticas indiretas, uma vez que ao se associarem com diferentes tipos de hemípteros, com a finalidade de protegê-los, as formigas podem espantar ou predar outros insetos e beneficiar indiretamente a planta hospedeira. Do ponto de vista do consumidor, qualidade e quantidade do recurso alimentar são de extrema importância para um forrageio ótimo. A variação e composição dos açúcares e aminoácidos que compõem o "honeydew" e o néctar extrafloral podem determinar a preferência das espécies de formigas por tais recursos. O "honeydew" e o néctar extrafloral podem conter aminoácidos similares em sua composição, porém o "honeydew" pode apresentar uma gama maior de açúcares em sua composição (melezitose, lactose, melibiose, maltose e rafinose) em comparação ao néctar extrafloral. No entanto, a composição dos mesmos pode variar de acordo com as espécies que os produzem. Assim, as fontes açucaradas são recursos que podem estar presentes na vegetação, tanto através do "honeydew", tanto através do néctar extrafloral e que podem estruturar e moldar a comunidade de insetos e particularmente das formigas arborícolas. As espécies pertencentes ao grupo polifilético das Poneromorfas são conhecidas por serem essencialmente carnívoras. Apesar disso, fontes de açúcar na vegetação,

MARQUES, Thamy Evellini Dias; CASTAÑO-MENESES, Gabriela; MARIANO, Cléa dos Santos Ferreira; DELABIE; Jacques H. C. Interações entre Poneromorfas e fontes de açúcar na vegetação. In: DELABIE, Jacques H. C. et al. As formigas poneromorfas do Brasil. Ilhéus: Editus, 2015. p. 361 -374. 
como néctar extrafloral e o "honeydew", também são importantes recursos para diversas espécies dessas formigas como, por exemplo, Ectatomma tuberculatum que pode obter de um terço à metade de seus recursos necessários em tais fontes açucaradas. Os gêneros Odontomachus e Ectatomma são os únicos entre as Poneromorfas que possuem espécies que realizam interações com hemípteros e utilizam o "honeydew" como recurso. Um número maior de táxons foi encontrado consumindo néctar extrafloral na literatura, como nos gêneros seguintes: Acanthoponera, Bothroponera, Diacamma, Gnamptogenys, Neoponera, Pachycondyla, Paraponera, Platythyrea e Rhytidoponera. Os recursos açucarados disponíveis na vegetação são considerados recursos-chave que favorecem a estruturação das comunidades de insetos arborícolas nas florestas tropicais, acarretando em forte influência dos mesmos na distribuição, densidade e diversidade de formigas. Como tais recursos são fontes ricas de carboidrato relativamente previsíveis, os mesmos podem ser de extrema importância até mesmo para diversas Poneromorfas que são principalmente carnívoras. Tal fato fica evidente através dos estudos aqui citados que relatam a interação entre diversas espécies de Poneromorfas e recursos açucarados na vegetação.

\section{Abstract}

Interaction between poneromorph ants and nectar sources in vegetation - Mutualism is an important ecological interaction in which both species are benefited by the association. This association can be obligatory or facultative, and is obligatory when the survival of at least one of the species involved depends on the association. Mutualistic associations can be further classified as direct and indirect. A direct mutualistic association occurs when there is direct physical contact, whereas in indirect mutualistic associations the species involved benefit without physical interaction. Mutualistic associations are extremely common among ants and plants, because these groups are highly diverse and abundant around the world. In general, when ants are involved in these interactions, the ants use resources in plants as nesting sites and/or food (e.g., honeydew or nectar) and, in return, the ants defend the plant against herbivores. Interactions between ants and extrafloral nectaries of plants exemplify direct mutualistic associations. The interactions between ant-plant-Hemiptera can exemplify indirect mutualistic interactions, since, by associating with Hemiptera, ants may scare away or prey on other insects and benefit the plant indirectly. From a consumer point of view, quality and quantity of food resources are extremely important for optimal foraging. The variation and composition of sugars and amino acids that make up the honeydew and extrafloral nectar can determine the preference of species of ants for such resources. The honeydew and extrafloral nectar may contain amino acids similar in composition, but the honeydew may have a wider range of sugars in its composition (e.g., melezitose, lactose, melibiose, maltose and raffinose) as compared to extrafloral nectar. However, the composition of such liquids may vary according to the species that produce them.

Thus, sugars are resources that may be present in the vegetation, both through the honeydew from hemipterans or through extrafloral nectar, and these can structure and shape the insect community, particularly that of arboreal ants. The species belonging to the polyphyletic poneromorphs are known to be essentially carnivores, however, the sugar sources such as extrafloral nectar and honeydew are also considered as important for some species such as ants (e.g. Ectatomma tuberculatum can consume one-third to half of its requirements from sugary sources). The genera Odontomachus and Ectatomma were thought to be unique amongst the poneropmorphs in having species involved in interactions with Hemiptera and using honeydew as a resource. However, a greater number of taxa have now been observed consuming extrafloral nectar, including members of the genera Acanthoponera, Bothroponera, Diacamma, Gnamptogenys, 
Neoponera, Pachycondyla, Paraponera, Platythyrea and Rhytidoponera. The available sugar resources in vegetation are considered key resources that favor the structuring of communities of arboreal insects in tropical forests, resulting in strong influence on the distribution, density and diversity of ants. Because such resources are rich sources of relatively predictable carbohydrates, they may be very important for several poneromorph species that have hitherto been considered to be mainly carnivorous. This fact is evident from the studies on poneromorphs cited in this chapter.

\section{Introdução}

Mutualismo é uma importante interação ecológica em que ambas as espécies associadas são beneficiadas (BOUCHER et al., 1982; BEATTIE, 1985; STADLER; DIXON, 2008). Tal associação pode ser obrigatória ou facultativa, sendo obrigatória quando a sobrevivência de pelo menos uma das espécies envolvidas depende da associação (BOUCHER et al., 1982; HÖLLDOBLER; WILSON, 1990). Associações mutualísticas podem ser classificadas, ainda, como diretas e indiretas. São consideradas como associações mutualísticas diretas quando as espécies da interação estão em contato físico diretamente; já nas associações mutualísticas indiretas, as espécies envolvidas na associação se beneficiam sem interagir fisicamente (BOUCHER et al., 1982).

Associações mutualísticas são extremamente comuns entre formigas e plantas, uma vez que tais agregações são abundantes e altamente diversas ao redor do mundo (HUXLEY; CUTLER, 1991). Em geral, nessas interações, as formigas encontram recursos nas plantas (locais de nidificação e alimento) e, em contrapartida, as formigas defendem a planta contra herbívoros (BENTLEY, 1977a; KOPTUR, 1979; SCHEMSKE, 1980; BEATTIE, 1985). O néctar produzido por nectários extraflorais representa um dos mais importantes recursos açucarados que as formigas podem encontrar em mais de 93 famílias de angiospermas e em algumas famílias de samambaias (KOPTUR, 1992).

Embora os nectários extraflorais (NEFs) sejam glândulas produtoras de néctar, elas não estão relacionadas ao processo de polinização e estão localizadas em diversas estruturas da planta tais como pecíolos, folhas, pedúnculos florais e frutos (KOPTUR, 1992). Outro recurso açucarado, denominado "honeydew", é amplamente consumido por inúmeras espécies de formigas e pode ser encontrado em plantas, porém de forma indireta (BUCKLEY, 1987). O "honeydew" é excretado pelos hemípteros após a metabolização da seiva elaborada que é extraída do floema de sua planta hospedeira (HUXLEY; CUTLER, 1991; BUCKLEY, 1987; DELABIE, 2001).

Dessa maneira, formigas e nectários extraflorais presentes em plantas exemplificam associações mutualísticas diretas (BUCKLEY, 1987). Nessas interações, as plantas fornecem glicose e aminoácidos através dos nectários extraflorais (NEFs), enquanto as formigas conferem proteção às plantas contra possíveis danos ocasionados por herbívoros (JANZEN, 1966; ELIAS; GELBAND, 1975). Já as interações entre formiga-hemíptero-planta podem exemplificar interações mutualísticas indiretas, uma vez que ao interagirem com subordens de hemípteros como Auchenorrhyncha (membracídeos, por exemplo) e Sternorrhyncha (afídeos, coccídeos e pseudococcídeos, por exemplo), as formigas podem beneficiar a planta indiretamente (DELABIE, 2001).

Através da interação formiga-planta-hemíptero, com a finalidade de proteger os hemípteros, as formigas podem espantar ou predar outros insetos (não hemípteros) que seriam danosos para a planta hospedeira e beneficiá-la indiretamente (BUCKLEY, 1987; DELABIE, 2001; STYRSKY; EUBANKS, 2007). Ampla gama de plantas, senão todas, pode abrigar os grupos na interação descrita acima, desde gramíneas (MOYA-RAYGOZA; NAULT, 2000) a arbustos e árvores (e. g. MOREIRA; DEL-CLARO, 2005), tanto em ambientes temperados, como em tropicais (WAY, 1963; BUCKLEY, 1987).

Tais associações são em sua maioria facultativas, sendo raras as associações mutualísticas obrigatórias, sugerindo que o mutualismo obrigatório raramente ocorre (RICO-GRAY, 1993). No 
entanto, entre as relações mutualísticas entre hemípteros e formigas, algumas espécies de hemípteros como o cicadelídeo mexicano Dalbulus quinquenotatus DeLong; Nault, não podem sobreviver e manter sua população na ausência de determinadas formigas (MOYA-RAYGOZA; NAULT, 2000).

Os NEFs geralmente são visitados por formigas não especialistas; no entanto, Janzen (1966) demonstrou que Pseudomyrmex ferrugineus Smith, 1877 é obrigatoriamente dependente dos recursos oferecidos pela espécie nativa da América central, Acacia cornigera (Fabaceae) Willd, enquanto a mesma é dependente da proteção de $P$. ferrugineus. Os NEFs presentes em A. cornigera constituem a principal e contínua fonte de açúcar de $P$. ferrugineus. Em contrapartida, $P$. ferrugineus é altamente agressiva e rápida, além de patrulhar $A$. cornigera durante 24 horas por dia, espantando e/ou predando insetos que seriam danosos para a planta.

Em florestas tropicais, a distribuição de formigas arborícolas e insetos no ambiente é bastante complexa, e a presença dos recursos representados por NEFs e "honeydew" é muitas vezes considerada como determinante para estruturar as comunidades (BLÜTHGEN et al., 2004). Na Amazônia venezuelana, Blüthgen et al. (2000) apontaram que as formigas amostradas utilizavam os dois tipos de recursos ao mesmo tempo, ou ao menos um, acarretando em forte influência dos mesmos na distribuição, densidade e diversidade de formigas arborícolas.

Através do efeito "bottom-up", a ocorrência de NEFs e "honeydew" pode contribuir para estruturar as comunidades (BLÜTHGEN et al., 2004). O "honeydew", no entanto, pode assumir um papel primordial na atuação do efeito "bottom-up", uma vez que pode estar sendo produzido em uma maior diversidade de espécies de plantas em relação aos NEFs (BLÜTHGEN et al. 2000; 2004). Além disso, espera-se que as formigas arborícolas dominantes irão competir pelo "honeydew", uma vez que o mesmo representa uma fonte de energia previsível dentro do território, e que seu controle acarreta interações antagonistas entre espécies ou talvez entre colônias (DAVIDSON; MCKEY, 1993).

Do ponto de vista do consumidor, qualidade e quantidade do recurso alimentar são de extrema importância para um forrageio ótimo (BLÜTHGEN et al., 2004). A variação e composição dos açúcares e aminoácidos que compõem o "honeydew" e o néctar podem determinar a preferência das espécies de formigas por tais recursos
(BLÜTHGEN; FIEDLER, 2004). O "honeydew" e o néctar podem conter aminoácidos similares em sua composição, porém o "honeydew" pode apresentar uma gama maior de açúcares (melezitose, lactose, melibiose, maltose e rafinose) em comparação ao néctar extrafloral (BLÜTHGEN et al., 2004). No entanto, a composição dos mesmos pode variar de acordo com as espécies que os produzem (COOK; DAVIDSON, 2006).

Assim, as fontes açucaradas são recursos previsíveis que podem estar presentes na vegetação, sob a forma de "honeydew" ou de néctar extrafloral, e que podem estruturar e moldar a comunidade de insetos e particularmente das formigas arborícolas (BLÜTHGEN et al., 2004). Essas fontes açucaradas podem ainda ser responsáveis pela manutenção da alta biomassa de formigas que ocorre nos dosséis tropicais, uma vez que somente o consumo de outros herbívoros poderia não ser o suficiente para manter tal elevada biomassa (DAVIDSON et al., 2003; WILSON; HÖLLDOBLER, 2005).

Por isso, ressaltamos a importância desses recursos, assim como as consequências ecológicas para as formigas (consumidor) e também para outros insetos que estão interagindo de forma direta e indireta e para a planta. Devido à previsibilidade desses recursos, os mesmos podem ser importantes também para grupos de formigas que são principalmente carnívoras, e destacamos a seguir como as formigas poneromorfas podem consumi-los.

\section{1) O consumo de honeydew e néctar por poneromorfas}

As espécies pertencentes ao grupo polifilético das poneromorfas são conhecidas por serem essencialmente carnívoras (HÖLLDOBLER; WILSON, 1990). No entanto, fontes açucaradas também podem ser consideradas importantes para algumas espécies dessas formigas, como por exemplo, Ectatomma tuberculatum (Olivier, 1792), que pode consumir de um terço à metade de suas necessidades nutricionais em fontes açucaradas como néctar extrafloral, "honeydew", e sob a forma de sementes coletadas no chão (DEJEAN; LACHAUD, 1992). Ectatomma edentatum Roger, 1863 foi apontada em alguns estudos por ser uma das espécies que se associam ao membracídeo Guayaquila xiphias (Fabricius, 1803) com maior frequência e consome "honeydew" (DEL-CLARO; OLIVEIRA, 1999; 2000). Odontomachus hastatus (Fabricius, 1804) 
é uma espécie frequentemente associada à epífita Philodendron insigne Schott (Araceae) e consumidora do néctar extrafloral produzido pela planta (GIBERNAU et al., 2007).

No entanto, o número de exemplos de interações como os apresentados acima entre poneromorfas, hemípteros e plantas que possuem nectários extraflorais é pequeno quando comparado com espécies de outras subfamílias tais como Myrmicinae, Formicinae e Dolichoderinae (RICO-GRAY; OLIVEIRA, 2007). Além disso, grande parte dos estudos que realizaram levantamentos de espécies que consomem líquidos açucarados ("honeydew" e néctar extrafloral) revelam que as poneromorfas estão em menor número quando comparadas às espécies de Formicinae e Myrmicinae (DEL-CLARO; OLIVEIRA, 1999; MOREIRA et al., 2000; BLÜTHGEN et al., 2004; MOREIRA; DEL-CLARO, 2005). Evans; Leston (1971) relatam que mesmo quando alguma poneromorfa, como Odontomachus troglodytes (Santschi, 1914), é encontrada protegendo hemípteros e se alimentando de "honeydew", a população de formigas é sempre pequena em comparação às Myrmicinae e Formicinae.

A história evolutiva das Formicidae que realizam interações com fontes de açúcar na vegetação fornece subsídios para melhor entender essas interações de acordo com cada subfamília (MOREAU et al., 2006). As poneromorfas são mais basais e tiveram sua radiação ao nível do solo da floresta e serapilheira, juntamente com o início da radiação das angiospermas (WILSON; HÖLLDOBLER, 2005). Posteriormente, as subfamílias Myrmicinae, Formicinae e Dolichoderinae tiveram uma radiação também em direção ao dossel da floresta concomitantemente com a expansão das angiospermas, modificando assim suas fontes alimentares como, por exemplo, adição de açúcares a sua dieta (WILSON; HÖLLDOBLER, 2005; RICO-GRAY; OLIVEIRA, 2007).

As espécies das subfamílias Formicinae e algumas de Dolichoderinae podem ser consideradas ainda como as mais especializadas dentro de Formicidae em consumir os líquidos açucarados (DAVIDSON, 1997; 1998; 2004). Isso se deve ao fato de que nessas espécies, o aparato digestivo sofreu modificações morfológicas permitindo que as mesmas estoquem uma maior quantidade de líquidos açucarados (EISNER; BROWN, 1957). O proventrículo, por exemplo, é uma estrutura digestiva que controla o fluxo de alimento entre o estômago social ("crop") e o estômago, e esse é rígido e esclerotizado somente nas espécies dessas famílias (EISNER; BROWN, 1957). O proventrículo esclerotizado permite uma estocagem e processamento de um volume superior de líquidos em relação às espécies que possuem um proventrículo primitivo, como no caso das poneromorfas (EISNER; BROWN, 1957; DAVIDSON, 1998). Como consequência da maior estocagem de líquidos açucarados, é possível que essas espécies possuam uma maior habilidade competitiva e habilidade exploratória nos dosséis tropicais (DAVIDSON et. al, 1998).

De forma geral, as poneromorfas apresentam uma relativa dificuldade em encontrar suas presas, principalmente quando tais espécies são especialistas (WILSON; HÖLLDOBLER, 2005). Fontes de açúcar na vegetação são consideradas mais previsíveis em relação a qualquer tipo de presa (DAVIDSON; MCKEY, 1993). Dessa maneira, as poneromorfas que utilizam os recursos açucarados, assim como as espécies pertencentes a outras subfamílias de Formicidae como Myrmicinae, Formicinae e Dolichoderinae, têm certa vantagem em relação às demais poneromorfas exclusivamente carnívoras.

\section{2) Interações entre NEFs, hemípteros e poneromorfas}

Os gêneros Odontomachus e Ectatomma são os únicos entre as poneromorfas que possuem espécies que realizam interações com hemípteros e utilizam o "honeydew" como recurso permanente. Já o néctar extrafloral é um recurso utilizado por um número maior de gêneros entre as poneromorfas, uma vez que além de Odontomachus e Ectatomma, também Neoponera, Paraponera, Acanthoponera, Diacamma, Rhytidoponera ou Gnamptogenys utilizam tal recurso. De forma geral, há um número maior de gêneros e espécies em Formicidae que consomem néctar extrafloral em comparação ao consumo de "honeydew", embora ambos sejam primordialmente fontes de açúcar (BLÜTHGEN et al., 2004). Dessa maneira, o número de espécies de formigas que utilizam "honeydew" pode representar apenas uma pequena fração de um número maior de espécies que utilizam néctar extrafloral dentro de uma comunidade (BLÜTHGEN et al., 2004).

Entre as poneromorfas, as espécies do gênero Ectatomma são as que mais utilizam fontes açucaradas como recurso, com cinco espécies 
documentadas que interagem com hemípteros e sete espécies que consomem néctar extrafloral (Tabelas 24.I e 24.II). Existe também um número maior de registros de Ectatomma utilizando fontes açucaradas, já que $76 \%$ das associações de hemípteros com poneromorfas são com Ectatomma. O mesmo padrão foi encontrado para as espécies de Ectatomma que consomem néctar extrafloral, já que $70 \%$ dos registros $(n=36)$ apontam esse gênero.

Embora as Ectatomma sejam predominantemente predadoras, algumas espécies como Ectatomma tuberculatum, Ectatomma ruidum (Roger, 1860), E. edentatum, E. brunneum (Fabricius, 1793) foram registradas utilizando recursos açucarados mais de uma vez e em diferentes países da região Neotropical (Tabelas 24.I e 24.II). Ectatomma edentatum, por exemplo, exibe um comportamento de forrageio diferenciado quando encontra gotas de "honeydew" no chão, uma vez que a mesma procura os hemípteros produtores desse recurso (DEL-CLARO; OLIVEIRA, 1996).

Apesar da excreção dos líquidos procedentes do metabolismo dos hemípteros sugadores de seiva corresponder a uma necessidade básica desses insetos, espalhar gotas de "honeydew" no solo e na vegetação pode também ser considerada uma estratégia para atrair formigas a fim de que estas sejam atendidas (DEL-CLARO; OLIVEIRA, 1996). Já E. ruidum exibe um comportamento especializado de forrageamento (tanto para escolha da fonte alimentar, quanto para o local do forrageamento) como coletoras de néctar extrafloral (SCHATZ et al., 1995).

Quanto às interações entre Odontomachus e hemípteros, quatro espécies desse gênero apresentam interações com hemípteros, e o mesmo número de espécies também consome néctar extrafloral (Tabelas 24.I e 24.II). O mais antigo registro de interação entre hemípteros e espécies de Odontomachus foi feito na ilha de Cuba entre Odontomachus insularis Guérin, 1844 e Mnemosyne cubana Stål, 1866 (Hemiptera: Cixiidae) (MYERS, 1929). No Brasil também há estudos relatando que algumas espécies de Odontomachus utilizam fontes açucaradas como recurso adicional como, por exemplo, O. haematodus (Linnaeus, 1758) e membracídeos em cacaueiros (DELABIE, 1990; MARQUES, Obs. Pess.).

$\mathrm{Na}$ Amazônia brasileira, cinco interações entre O. bauri Emery, 1892 e ninfas de Xedreota tuberculata (Osborn, 1938) foram observadas
(SOUZA; FRANCINE, 2010). Em cada observação, foram encontrados de dois a quatro indivíduos de O. bauri em atividades diurnas e noturnas. Para O. bauri solicitar o "honeydew", a mesma usou as antenas para pressionar a região inferior do abdômen do hemíptero, se alimentando diretamente do orifício anal de $X$. tuberculata, com as mandíbulas abertas (SOUZA; FRANCINE, 2010). O mecanismo de pressionar o abdômen do hemíptero para facilitar a ejeção do "honeydew" é comumente observado nesse tipo de interação (WAY, 1963). Além das antenas para pressionar o abdômen do hemíptero, as formigas também podem usar as pernas para pressioná-lo ou simplesmente balançá-lo (WAY, 1963).

Fora da Região Neotropical, outro registro do gênero em interação foi documentado entre O. troglodytes Santschi, 1914 com afídeos da espécie Toxoptera aurantii (Boyer De Fonscolombe, 1841) e coccídeos da espécie Stichococcus sjostedti Cockerell, 1903 (EVANS; LESTON, 1971). Para a proteção dos hemípteros, as formigas construíram "tetos" ou abrigos a partir de partículas de solo. De fato, é comum formigas arborícolas construírem abrigos com restos vegetais para proteger seus hemípteros mutualísticos contra predadores e chuva. (DELABIE, 2001). É bem provável que esses tetos também contribuem a evitar a fuga das espécies de hemípteros móveis, em particular os Membracidae.

Espécies diferentes de Odontomachus que se associam a hemípteros, como O. hastatus, OdontomachusruficepsSmith, 1858, Odontomachus brunneus (Patton, 1894) e Odontomachus laticeps Roger, 1861, foram registradas consumindo néctar extrafloral (BLÜTHGEN; FIEDLER, 2004; GIBERNAU et al., 2007). A epífita Philodendron insigne se associa facultativamente a várias espécies de formigas arborícolas oportunistas. No entanto, a espécie que é mais eficaz na proteção de $P$. insigne é $O$. hastatus, que é a inquilina predominante, sugerindo estreita associação entre essas espécies (GIBERNAU et al., 2007). O. brunneus e O. laticeps foram encontradas associadas a outra epífita, Caularthron bilamellatum (Rchb. f.) R.E. Schult (Orchidaceae) (FISHER; ZIMMERMAN, 1988). No entanto, essa última associação foi apontada por ser generalista, ao contrário da interação descrita anteriormente.

O número de outras espécies e gêneros de poneromorfas que interagem com recursos açucarados na vegetação é pequeno, embora espécies 
de Neoponera também tenham sido muito documentadas interagindo com nectários extraflorais. No entanto, outros gêneros/espécies como no caso de Diacamma sp., Rhytidoponera spoliata (Emery, 1895), Gnamptogenys semiferox Brown, 1958 e Bothroponera tesseronoda (Emery, 1877) foram raramente documentados e somente interagindo com nectários extraflorais (Tabela 24.II).

Assim, as espécies pertencentes ao grupo das poneromorfas, embora preferencialmente predadoras, também podem exibir comportamento de forrageio diferenciado quando buscam ou encontram fontes açucaradas como recurso. Além disso, também podem exibir comportamentos semelhantes às das subfamílias que são mais especializadas na busca e no uso desses recursos, tais como Myrmicinae, Formicinae ou Dolichoderinae.

\section{3) Inferências ecológicas}

Os hemípteros podem causar danos às plantas hospedeiras, no entanto, quando estão associados às formigas, essa interação pode ser benéfica às plantas (BUCKLEY, 1987; WAY; KHOO, 1992; STYRSKY; EUBANKS, 2007). Com a finalidade de proteger os hemípteros, as formigas podem atacar, predar ou dificultar a atividade de outros herbívoros, beneficiando a planta indiretamente (EUBANKS; STYRSKY, 2006; PERFECTO; VANDERMEER, 2006). Embora existam relatos de que essas interações sejam prejudiciais para a planta, $73 \%$ dos estudos revistos por Styrsky; Eubanks (2007) relatam consequências positivas para a planta hospedeira decorrentes das interações entre formigas e hemípteros.

TABELA 24.I - Resumo de 22 estudos de interações entre poneromorfas e hemípteros e as localidades de ocorrência das interações. 1, Myers (1929); 2, Evans; Leston (1971); 3, Souza; Francini (2010); 4, Delabie (1990); 5, Marques et al. obs. pess; 6, Weber (1946); 7, Lachaud et al. (1982); 8, Lachaud (1982); 9, Pratt (1989); 10, Lachaud (1990); 11, García-Pérez et al. (1991); 12, Dejean; Lachaud (1992); 13, Valenzuela-González et al. (1995); 14, Moreira et al. 2000; 15, Del-Claro; Oliveira (1996); 16, Del-Claro; Oliveira (1999); 17, Del-Claro; Oliveira (2000); 18, Moreira; Del-Claro (2005); 19, Del-Claro et al. (2006); 20, Bächtold; Del-Claro (2013); 21, Rodrigues; Cassino (2011); 22, Campos; Camacho (2014).

\begin{tabular}{|c|c|c|c|c|c|}
\hline Subfamília & Espécies de formigas & Espécies de hemípteros & Família & Localidade & Referência \\
\hline \multirow[t]{12}{*}{ Ectatomminae } & Ectatomma brunneum & Guayaquila xiphias & Membracidae & Brasil & 21 \\
\hline & Ectatomma brunneum & Enchenopa brasiliensis & Membracidae & Brasil & $14,18,19$ \\
\hline & Ectatomma edentatum & Guayaquila xiphias & Membracidae & Brasil & $15,16,17$ \\
\hline & $\begin{array}{l}\text { Ectatomma ruidum } \\
\text { Ectatomma ruidum }\end{array}$ & $\begin{array}{l}\text { Membracídeos sem } \\
\text { identificação } \\
\text { Hemípteros sem } \\
\text { identificação }\end{array}$ & & $\begin{array}{l}\text { USA, México } \\
\text { Panamá }\end{array}$ & $\begin{array}{l}6,7 \\
9\end{array}$ \\
\hline & Ectatomma ruidum & $\begin{array}{l}\text { Hemípteros sem } \\
\text { identificação }\end{array}$ & & México & 10 \\
\hline & Ectatomma tuberculatum & $\begin{array}{l}\text { Hemípteros sem } \\
\text { identificação }\end{array}$ & & México & 11 \\
\hline & Ectatomma tuberculatum & $\begin{array}{l}\text { Membracídeos sem } \\
\text { identificação }\end{array}$ & & Brasil, USA & 5,6 \\
\hline & Ectatomma tuberculatum & & & Brasil, México, & $5,8,12$ \\
\hline & & Aphis gossypii & & Brasil & \\
\hline & Ectatomma tuberculatum & & & Bracil & \\
\hline & Ectatomma tuberculatum & $\begin{array}{l}\text { Hemípteros sem } \\
\text { identificação }\end{array}$ & Aphididae & Brasil & $\begin{array}{l}20 \\
22\end{array}$ \\
\hline & Odontomachus bauri & Xedreota tuberculata & Cicadellidae & Brasil & 3 \\
\hline \multirow[t]{4}{*}{ Ponerinae } & $\begin{array}{l}\text { Odontomachus } \\
\text { haematodus }\end{array}$ & $\begin{array}{l}\text { Membracídeos sem } \\
\text { identificação }\end{array}$ & & Brasil & 4,5 \\
\hline & Odontomachus insularis & Mnemosyne cubana & Cixiidae & Cuba & 1 \\
\hline & $\begin{array}{l}\text { Odontomachus } \\
\text { troglodytes }\end{array}$ & Toxoptera aurantii & Aphididae & Gana & 2 \\
\hline & $\begin{array}{l}\text { Odontomachus } \\
\text { troglodytes }\end{array}$ & Stictococcid sjostedti & Coccidae & Gana & 2 \\
\hline
\end{tabular}


TABELA 24.II - Resumo de 42 estudos descrevendo ou notificando interações entre poneromorfas e nectários extraflorais e localidades de ocorrência das interações. 1, Cook (1904,1905); 2, Koptur (1984); 3, Bentley (1977a); 4, Bentley (1976); 5, Bentley (1977b); 6, Schemske (1980); 7, Fisher; Zimmerman (1988); 8, Devries (1991); 9, Oliveira; Pie (1998); 10, Rickson; Rickson (1998); 11, De la Fuente; Marquis (1999); 12, Schemske (1982); 13, Bennett; Breed (1985); 14, Altshuler (1999); 15, Del-Claro et al. 1996; 16, Pratt (1989); 17, Passera et al. 1994; 18, Agarwal; Rastogi (2009); 19, Pérez-Lachaud et al. (2006); 20, Oliveira; Freitas (2004); 21, Blüthgen et al. (2004); 22, Santos; Del-Claro (2001); 23, Gibernau et al. (2007); 24, Guimarães Jr. et al. (2006); 25, Mondal et al. (2013); 26, Lange et al. (2009); 27, Dáttilo et al. (2014); 28, Apple; Feener (2001); 29, Korndörfer; Del-Claro (2006); 30, Nascimento; Del-Claro (2010); 31, Byk; Del-Claro (2010); 32, Nahas; Del-Claro (2011); 33, Assunção et al. (2014); 34, Assunção et al. (2014); 35, Vilela et al. (2014); 36, Valenzuela-González et al. (1995); 37, Schatz et al. (1995); 38, Weber (1946); 39, Costa et al. (1992); 40, Horvitz; Schemske (1984); 41, Jaffe et al. (1989); 42, Dejean; Suzzoni (1997); 43, Campos; Camacho (2014).

\begin{tabular}{|c|c|c|c|}
\hline Subfamília & Espécies de formigas & Localidade & Referência \\
\hline Heteroponerinae & Acanthoponera sp. & Brasil & 9 \\
\hline \multirow[t]{20}{*}{ Ectatomminae } & Ectatomma tuberculatum & Estados Unidos & $1,26,36$ \\
\hline & Ectatomma tuberculatum & Costa Rica & $2,3,4,5,28$ \\
\hline & Ectatomma tuberculatum & Panamá & $7,8,11,12$ \\
\hline & Ectatomma tuberculatum & Brasil & $\begin{array}{l}22,27,30,31,32, \\
33,34,35,43\end{array}$ \\
\hline & Ectatomma tuberculatum & Estados Unidos & 38 \\
\hline & Ectatomma brunneum & Brasil & $22,24,39$ \\
\hline & Ectatomma ruidum & Experimental & 6,37 \\
\hline & Ectatomma ruidum & Panamá & $7,8,12,16$ \\
\hline & Ectatomma ruidum & México & 17,19 \\
\hline & Ectatomma ruidum & Costa Rica & 28 \\
\hline & Ectatomma ruidum & México & 41 \\
\hline & Ectatomma permagnum & Brasil & $15,30,31$ \\
\hline & Ectatomma edentatum & Brasil & $20,32,33$ \\
\hline & Ectatomma edentatum & México & 26 \\
\hline & Ectatomma planidens & México & 26 \\
\hline & Ectatomma planidens & Brasil & 30,33 \\
\hline & Ectatomma opaciventre & Brasil & 30,31 \\
\hline & Ectatomma sp. & Brasil & $9,14,22,29,35$ \\
\hline & Rhytidoponera spoliata & Austrália & 21 \\
\hline & Gnamptogenys semiferox & Brasil & 35 \\
\hline \multirow[t]{15}{*}{ Ponerinae } & Bothroponera tesseronoda & Índia & 18 \\
\hline & Diacamma sp. & Sri Lanka, Índia, Malásia & 10 \\
\hline & Neoponera villosa & México & 26 \\
\hline & Neoponera villosa & Brasil & $22,31,34$ \\
\hline & Neoponera foetida & Brasil & 31 \\
\hline & Neoponera obscuricornis & Brasil & 31 \\
\hline & Neoponera unidentata & México & 40 \\
\hline & Neoponerasp. & Costa Rica & 5 \\
\hline & Neoponerasp. & Panamá & 8 \\
\hline & Neoponera sp. & Índia & 25 \\
\hline & Odontomachus brunneus & Panamá & 7 \\
\hline & Odontomachus hastatus & Guiana Francesa & 23 \\
\hline & Odontomachus laticeps & Panamá & 7 \\
\hline & Odontomachus ruficeps & Austrália & 21 \\
\hline & Pachycondyla striata & Brasil & 31 \\
\hline Ponerinae & Platythyrea conradti & México (em laboratório) & 42 \\
\hline Paraponerinae & Paraponera clavata & Costa Rica & 13 \\
\hline
\end{tabular}


Inúmeros trabalhos apontam que as formigas presentes em tais interações podem ser eficazes para realizar controle biológico em sistemas agrícolas (KHOO; HO, 1992; VANDERMEER, et al., 2002; EUBANKS; STYRSKY, 2006; PERFECTO; VANDERMEER 2006; JHA et al., 2012), já que as formigas podem predar ou reduzir a atividade de outros herbívoros supostamente mais prejudiciais para o "fitness" das plantas (VANDERMEER, et al., 2002). Dessa maneira, a ocorrência das interações entre formiga -hemíptero pode acarretar em efeitos negativos na densidade e riqueza de espécies de vários insetos herbívoros, assim como efeitos positivos na densidade dos hemípteros associados (STYRSKY; EUBANKS, 2007). Os efeitos benéficos que as plantas podem obter a partir da interação formiga-hemíptero também ocorrem quando as formigas se associam a nectários extraflorais, uma vez que elas podem matar ou perturbar a atividade dos herbívoros que se aproximarem da planta (RICO-GRAY; OLIVEIRA, 2007).

Quanto às consequências a partir das interações de nectários extraflorais, hemípteros e poneromorfas, o gênero que se destaca é Ectatomma. No início do século XX, E. tubercutalum foi observada interagindo com nectários extraflorais do algodão (Gossypium spp., Malvaceae), causando um decréscimo nas populações de herbívoros (larvas de $A n$ thonomus grandis Boheman, 1843, Curculionidae) danosos à planta (COOK, 1904; 1905). A partir desse registro, a espécie foi indicada para ser usada num dos mais antigos programas de controle biológico dos Estados Unidos (COOK, 1904; 1905). E. ruidum também foi sugerida para seu uso em programa de controle biológico a partir de observações realizadas sobre a interação com hemípteros e nectários extraflorais em cultivo de algodão e citros (WEBER, 1946).

Alguns estudos no Cerrado do Brasil mostram uma rede de benefícios entre algumas espécies de Ectatomma (assim como outras espécies de formigas), plantas e hemípteros (quando presentes em interação) e decréscimo na abundância de alguns herbívoros (DEL-CLARO et al., 2006). No geral, existem poucos estudos que discorram sobre as consequências ecológicas envolvendo interações entre as espécies de formigas e fontes açucaradas (EUBANKS; STYRSKY, 2006; RICO-GRAY; OLIVEIRA, 2007) e essas consequências ecológicas precisam ser investigadas.

\section{4) Transmissão de líquidos entre poneromorfas}

As subfamílias mais derivadas da família Formicidae (e.g. Formicinae e Dolichoderinae) compartilham alimentos líquidos entre indivíduos da mesma espécie, onde o recurso coletado por poucos indivíduos pode ser consumido por muitos, através da trofalaxia (DEJEAN; SUZZONI, 1997). Tal processo ocorre quando alimentos líquidos (e.g. "honeydew" e néctar extrafloral) são regurgitados para permitir que vários outros membros da colônia se alimentem, após inicialmente ser estocados em seu "estômago social" (crop) (HÖLLDOBLER, 1985).

As poneromorfas não possuem estômago social, mas podem ser consideradas como precursoras no processo evolutivo para o comportamento especializado de transporte de líquidos (HÖLLDOBLER; WILSON, 1990). Essa afirmação é devida ao fato de que várias espécies do grupo podem transportar líquidos por diversas estratégias, muitas vezes denominadas pseudotrofalaxia, já que não há regurgitação (LACHAUD; DEJEAN, 1991).

As espécies E. tuberculatum e E. ruidum transportam líquidos açucarados como "honeydew" e néctar extrafloral com o auxílio de suas mandíbulas (WEBER, 1946). Nesse caso, o líquido é retido na forma de uma gota pela ação conjugada da tensão superficial (que mantém a coesão do líquido) e da capilaridade (que a mantém agregada aos pelos e asperezas da região oral do inseto). Outras espécies como Paraponera clavata (Fabricius, 1775) (MCCLUSKEY; BROWN, 1972; HERMANN, 1975), Odontomachus troglodytes (EVAN; LESTON, 1971; LACHAUD; DEJEAN, 1991), Neoponera verenae Forel, 1922 e Neoponera villosa, Hypoponera sp. (HASHIMOTO et al., 1995), Ponera coarctata (Latreille, 1802) (LIEBIG et al., 1997) e Platythyrea conradti Emery, 1899 (DEJEAN; SUZZONI, 1997) também foram observadas transportando líquidos da mesma maneira.

Para acumular os líquidos açucarados em suas mandíbulas, as espécies citadas acima "lambem” sucessivamente tais líquidos, ao contrário de espécies pertencentes a outras subfamílias mais derivadas, que sugam os líquidos (PAUL; ROCES, 2003). Essa diferença no modo de forrageamento pode ser o reflexo da ausência de estômago social nas poneromorfas, que precisam acumular as gotas em suas mandíbulas e lamber, o que pode ser uma melhor estratégia em relação à sucção dos líquidos (PAUL; ROCES, 2003).

No caso das espécies citadas acima, o transporte de líquidos presos às mandíbulas e o compartilhamento através de pequenas porções para os outros indivíduos são conhecidos como "social 
bucket" (HÖLLDOBLER, 1985; HÖLLDOBLER; WILSON, 1990). Precedendo o compartilhamento de líquidos, a comunicação entre os indivíduos (e.g necessidade de alimento da colônia) pode ser realizada através de sinais de antenação entre as formigas (batidas mútuas usando as antenas) (HÖLLDOBLER, 1985).

A comunicação e compartilhamento de líquidos descritos a partir de observações de $O$. troglodytes revelaram que, após a mútua antenação, a gota de líquido presa entre as mandíbulas era transferida para as pernas traseiras e compartilhada entre os indivíduos da colônia através de pseudotrofalaxia (LACHAUD; DEJEAN, 1991). Outra adaptação para o transporte de líquidos açucarados foi encontrada em $P$. conradti, onde esses líquidos ficam presos às mandíbulas sob força da tensão superficial, o néctar extrafloral se fixando entre a cabeça, tórax e pernas anteriores (DEJEAN; SUZZONI, 1997).

\section{Conclusões}

A história evolutiva dos parceiros das interações descritas, tanto das formigas, quanto das angiospermas e dos hemípteros, conta muito sobre as atuais interações entre formiga-planta e o papel dos recursos disponíveis na planta. Dessa maneira, as subfamílias Myrmicinae, Formicinae e Dolichoderinae tiveram sua radiação a partir do solo e da serapilheira em direção ao dossel da floresta, acompanhando, de certa forma, a expansão das angiospermas e dos insetos herbívoros, tais como os hemípteros (WILSON; HÖLLDOBLER, 2005; MOREAU, et al., 2006). Em contrapartida, as poneromorfas tiveram sua radiação praticamente limitada ao chão (WILSON; HÖLLDOBLER, 2005). Esses fatos podem ter resultado na maior especialização de poucas poneromorfas nas interações com plantas e recursos açucarados disponíveis na vegetação.

Os recursos açucarados disponíveis na vegetação são considerados recursos-chave que favorecem a estruturação das comunidades de insetos arborícolas nas florestas tropicais, acarretando em forte influência dos mesmos na distribuição, densidade e diversidade de formigas. Como tais recursos são fontes ricas em carboidrato e relativamente previsíveis, os mesmos podem ser de extrema importância também para diversas poneromorfas predominantemente carnívoras. Um número maior de espécies dessas formigas que consomem néctar extrafloral foi encontrado em relação ao consumo do "honeydew", sendo que este padrão também se estende a todas as demais subfamílias de formigas que utilizam recursos açucarados como fontes nutricionais.

$\mathrm{O}$ fato de que poneromorfas sejam essencialmente carnívoras pode acarretar ainda na raridade de estudos sobre o forrageio dessas formigas, resultando no baixo número de estudos em comparação às formigas onívoras. Assim, um maior número de estudos sobre as interações entre fontes açucaradas disponíveis na vegetação e as poneromorfas poderia revelar uma maior diversidade dessas formigas que também podem utilizar esses recursos. Mais investigações são necessárias para demonstrar a importância ecológica desses recursos açucarados para formigas predominantemente carnívoras, tais como as poneromorfas.

\section{Referências}

AGARWAL, V. M.; RASTOGI, N. Spatio temporal dynamics and plant-part preference patterns of the plant-visiting ants and the insect herbivores of sponge gourd plants. Journal of Asia-Pacific Entomology, v. 12, p. 57-66, 2009.

APPLE, J. L.; FEENER, D. H. JR. Ant visitation of extrafloral nectaries of Passiflora: the effects of nectary attributes and ant behavior on patterns in facultative antplant mutualisms. Oecologia, v. 127, p. 409-416, 2001.

ALTSHULER, D. L. Novel interactions of nonpollinating ants with pollinators and fruit consumers in a tropical forest. Oecologia, v. 119, p. 600-606, 1999.

ASSUNÇÃO, M. A.; TOREZAN-SILINGARDI, H. M.; DEL-CLARO, K. Do ant visitors to extrafloral nectaries of plants repel pollinators and cause an indirect cost of mutualism? Flora, v. 209, p. 244-249, 2014.

BÄCHTOLD, A.; DEL-CLARO, K. Predatory behavior of Pseudodorus clavatus (Diptera, Syrphidae) on aphids tended by ants. Revista Brasileira de Entomologia, v. 57, n. 4, p. 437-439, 2013.

BEATTIE, A. J. The Evolutionary Ecology of Antplant Mutualisms. Cambridge University Press, 1985.

BENNETT, B.; BREED, M. D. On the association between Pentaclethra macroloba (Mimosaceae) and Paraponera clavata (Hymenoptera: Formicidae) colonies. Biotropica, v. 17, p. 253-255, 1985.

BENTLEY, B. L. Plants bearing extrafloral nectaries and the associated ant community: Interhabitat differences in the reduction of herbivore damage. Ecology, v. 57, p. 815-820, 1976. 
BENTLEY, B. L. Extrafloral nectaries and pugnacious bodyguards. Annual Review of Ecology and Systematics, v. 8, p. 407-427, 1977a.

BENTLEY, B. L. The protective function of ants visiting the extrafloral nectarines ồ Bixa orellana (Bixaceae). Journal of Ecology, v. 65, p. 27-38, 1977b.

BOLTON, B. Synopsis and classification of Formicidae. Memoirs of the American Entomologic Institute v. 71, p. 370, 2003.

BOUCHER, D. H.; JAMES, S.; KEELER, K. H. The ecology of mutualism. Annual Review of Ecology and Systematics, 13:315-47, 1982.

BLÜTHGEN, N.; FIEDLER, K. Preferences for sugars and amino acids and their conditionality in a diverse nectar-feeding ant community. Journal of Animal Ecology, v. 73, p. 155-166, 2004.

BLÜTHGEN, N.; STORK, E. N.; FIEDLER, K. Bottom-up control and co-occurrence in complex communities: honeydew and nectar determine a rainforest ant mosaic. Oikos, 106: 344-358, 2004.

BLÜTHGEN, N. E. S.; VERHAAGH, M.; GOITÍA, W.; JAFFÉ, K.; MORAWETZ, W.; BARTHLOTT, W. How plants shape the ant community in the Amazonian rainforest canopy: the key role of extrafloral nectaries and homopteran honeydew. Oecologia, v. 125, p. 229240, 2000.

BUCKLEY, R. C. Interactions involving plants, Homoptera, and ants. Annual Review of Ecology and Systematics, v. 18, p. 111-135, 1987.

BYK, J.; DEL-CLARO, K. Nectar- and pollengathering Cephalotes ants provide no protection against herbivory: a new manipulative experiment to test ant protective capabilities. Acta Ethologica, v. 13, p. 33-38, 2010.

CAMPOS, R. I.; CAMACHO, G. P. Ant-plant interactions: the importance of extrafloral nectaries versus hemipteran honeydew on plant defense against herbivores. Arthropod-Plant Interactions, v. 8, p. 507-512, 2014.

COOK, O. F. Report on habits of kelep, or Guatemalan cotton-boll-weevil ant. Bureau of Entomology Bulletin, n.s., 49. Washington, DC: Government Printing Office, 1904.

COOK, O. F. Social organization and breeding habits of cotton-protecting kelep of Guatemala. Bureau of Entomology, Technical Series 10. Washington, DC: Government Printing Office, 1905.
COOK, S. C.; DAVIDSON, D. W. Nutritional and functional biology of exudate feeding ants. Entomologia Experimentalis et Applicata, v. 118, p. 1-10, 2006.

COSTA, F. M. C. B.; OLIVEIRA-FILHO, A. T.; OLIVEIRA, P. S. The role of extrafloral nectaries in Qualea grandiflora (Vochysiaceae) in limiting herbivory: an experiment of ant protection in cerrado vegetation. Ecological Entomology, v. 17, p. 363-365, 1992.

DÁTTILO, W.; FAGUNDES, R.; GURKA, C. A. Q.; SILVA, M. S. A.; VIEIRA, M. C. L.; IZZO, T. J.; DÍAZCASTELAZO, C.; DEL-CLARO, K.; RICO-GRAY. V. Individual-Based Ant-Plant Networks: DiurnalNocturnal Structure and Species-Area Relationship. PLoS One, v. 9, n. 6, e99838, 2014.

DAVIDSON, D. W. The role of resource imbalances in the evolutionary ecology of tropical arboreal ants. Biological Journal of the Linnean Society, v. 61, p.153-181, 1997.

DAVIDSON, D. W.; COOK, S. C.; SNELLING, R. R.; CHUA, T. H. Explaining the abundance of ants in lowland tropical rainforest canopies. Science, v. 300, p. 969-972, 2003.

DAVIDSON, D. W.; COOK, S. C.; SNELLING, R. R. Liquid-feeding performances of ants (Formicidae): Ecological and evolutionary implications. Oecologia, v. 139, p. 255-266, 2004.

DEJEAN, A.; LACHAUD, J. P. Growth-related changes in predation behavior in incipient colonies of the ponerine ant Ectatomma tuberculatum (Olivier). Insectes Sociaux, v. 39, n. 2, p. 129-143, 1992.

DEJEAN, A.; SUZZONI, J. P. Surface tension strengths in the service of a ponerine ant: A new kind of nectar transport. Naturwissenschaften, v. 84, p. 76-79. 1997.

DELABIE, J. H. C. The ant problems of cocoa farms in Brazil,. In R.K. Vander Meer, K. Jaffe; A. Cedeño (eds.), Applied Myrmecology: A World Perspective, p. 555569, 1990.

DELABIE, J. H. C. Trophobiosis between Formicidae and Hemiptera (Sternorrhyncha and Auchenorrhyncha): An overview. Neotropical Entomology, v. 30, p. 501-16, 2001.

DE LA FUENTE, M. A. S.; MARQUIS, R. J. The role of ant-tended extrafloral nectaries in the protection and benefit of a Neotropical rainforest tree. Oecologia, v. 118, p. 192-202, 1999.

DEL-CLARO, K.; OLIVEIRA, P. S. Honeydew flicking by treehoppers provides cues to potential tending ants. Animal Behavior, v. 51, p. 1071-1075, 1996. 
DEL-CLARO, K.; OLIVEIRA, P. S. Ant-Homoptera interactions in a Neotropical savanna: the honeydew-producing treehopper Guayaquila xiphias (Membracidae) and its associated ant fauna on Didymopanax vinosum (Araliaceae). Biotropica, v. 31, n. 1, p. 135-144, 1999.

DEL-CLARO, K.; OLIVEIRA, P. S. Conditional outcomes in a neotropical treehopper-ant association: temporal and species-specific variation in ant protection and homopteran fecundity. Oecologia, v. 124, p.156-165, 2000.

DEL-CLARO, K.; BERTO, V.; RÉU, W. Effect of Herbivore Deterrence by Ants on the Fruit Set of an Extrafloral Nectary Plant, Qualea multiflora (Vochysiaceae). Journal of Tropical Ecology, Aberdeen, v.12, p. 887-892, 1996.

DEL-CLARO, K.; BYK, J.; YUGUE, G. M.; MORATO, M. G. Conservative benefits in an anthemipteran association in the Brazilian tropical savanna. Sociobiology, v. 47, n. 2, p. 415-421, 2006.

DEVRIES, P. J. Mutualism between Thisbeirenea butterflies and ants, and the role of ant ecology in the evolution of larval-ant associations. Biological Journal of the Linnean Society, v. 43, p 179-195, 1991.

ELIAS, T. S.; GELBAND, H. Nectar: Its production and function in trumpet creeper. Science, v. 189, p. 289$291,1975$.

EUBANKS, M. D.; STYRSKY, J. D. Ant-hemipteran mutualisms: keystone interactions that alter food web dynamics and influence plant fitness. Trophic and Guild Interactions in Biological Control, v. 3, p. 171-189, 2006.

EVANS, H. C.; LESTON, D. A ponerinae ant (Hymenoptera, Formicidae) associated with homoptera on cocoa in Ghana. Bulletin

Entomological Research, v. 61, p. 357-361, 1971.

GARCÍA-PÉREZ, J. A.; PEÑA-SANCHES, R.; CAMARGO-HUIQUI, P.; CHAMPALBERT, A. Rutas de forrajeo utilizadas por Ectatomma tuberculatum O. (Hymenoptera, Ponerinae) em una plantación de cacao em El Sononusco, Chiapas, Mexico. Folia Entomologica Mexicana, v. 82, p. 161-171, 1991.

FISHER, B. L.; ZIMMERMAN, J. K. Ant/orchid associations in the Barro Colorado National Monument, Panama. Lindleyana v. 3, p. 12-16, 1988.

GIBERNAU, M.; ORIVEL, J.; DELABIE, J. H. C.; BARABE, D.; DEJEAN, A. An asymmetrical relationship between an arboreal ponerine ant and a trash-basket epiphyte (Araceae). Biological Journal of the Linnean Society, v. 91, p 341-346, 2007.
GUIMARÃES, J. R. P. R.; RAIMUNDO, R. L. G.; BOTTCHER, C.; SILVA, R. R.; TRIGO, J. R. Extrafloral nectaries as a deterrent mechanism against seed predators in the chemically protected weed Crotalaria pallida (Leguminosae). Austral Ecology, v. 31, p. 776-782, 2006.

HASHIMOTO, Y.; YAMAUCHI, K.; HASEGAWA, E. Unique habits of stomodeal trophallaxis in the ponerine ant Hypoponera sp. Insectes Sociaux, v. 42, p. 137-144, 1995.

HERMANN, H. R. Crepuscular and nocturnal activities of Paraponera clavata (Hymenoptera: Formicidae: Ponerinae). Entomological News, v. 86, p. 94-98. 1975.

HÖLLDOBLER, B. Liquid food transmission and antennation signals in ponerinae ants. Israelian Journal of Entomology, 19: 89-99. 1985.

HÖLLDOBLER, B.; WILSON, E. O. The Ants. Cambridge, M A: Belknap Press of Harvard University Press. 1990.

HORVITZ, C. C.; SCHEMSKE, D. W. Effects of ants and an ant-tended herbivore on seed production of a Neotropical herb. Ecology, v. 65, p. 1369-1378, 1984.

HUXLEY, C. R.; CUTLER, D. F. Ant-plant

interactions. Oxford: Oxford University Press. 1991.

JAFFE, K.; PAVIS, C.; VANSUYT, G.; KERMARREC, A. Ants visit extrafloral nectaries of the orchid Spatho glottis plicata Blume. Biotropica, v. 21, p. 278-79, 1989.

JANZEN, D. H. Coevolution of mutualism between ants and acacias in Central America. Evolution, v. 20, p. 249-275. 1966.

JHA, S.; ALLEN, D.; LIERE, H.; VANDERMEER J.; PERFECTO, I. Mutualisms and Population Regulation: Mechanism Matters. Plos One, v. 7, n. 8: e43510, 2012.

KHOO, K. C.; HO, C. T. The influence of Dolichoderus thoracicus (Hymenoptera: Formicidae) on losses due to Helopelitis theivora (Heteroptera: Miridae), black poddisease, and mammalian pests in cocoa in Malaysia. Bulletin Entomological Research, v. 82, p. 485-491, 1992.

KOPTUR, S. Facultative mutualism between weedy vetches bearing extrafloral nectaries and weedy ants I California. American Journal of Botany, v. 66, p. 10161020, 1979.

KOPTUR, S. Experimental Evidence for Defense of Inga (Mimosoideae). Ecology, v. 65, n. 6: 1787-1793, 1984.

KOPTUR, S. Extrafloral nectary-mediated interactions between insects and plants. In Insect-Plant Interactions, ed. E. Bernays, 4:81-129. Boca Raton,FL: CRC Press. 1992. 
KORNDÖRFER, A. P.; DEL-CLARO, K. Ant defense versus induced defense in Lafoensia pacari (Lythraceae), a myrmecophilous tree of the Brazilian cerrado. Biotropica, v. 38, n. 6, p. 786-788. 2006.

LACHAUD, J. P. Las presas de Ectatomma tuberculatum (Roger) sobre plantas de café y cacao em El Soconusco, Chis (Hymenoptera: Formicidae). In: CONGRESSO NACIONAL DE ENTOMOLOGIA, 54, 1982, Chiapas. Resumos...Chiapas: Folia Entomológica Mexicana, 1982. p. 76-83.

LACHAUD, J. P. Foraging activities in some Neotropical Ponerinae ants Ectatomma ruidum (Hymenoptera: Formicidae). Folia Entomológica Mexicana, v. 78, p. 241-256, 1990.

LACHAUD, J. P.; FRESNEAU, D.; GARCÍA-PÉREZ, J.A. Estude des stratégies d'approvisionnement chez trois espécies de fourmis ponerines. Folia Entomologica Mexicana, v. 61, p. 159-177, 1982.

LIEBIG, J.; HEINZE, J.; HÖLLDOBLER, B.

Trophallaxis and aggression in the ponerine ant, Ponera coarctata: Implications for the evolution of liquid food exchange in the Hymenoptera. Ethology, v. 103, p.707-722. 1997.

LANGE, D.; DÁTTILO, W.; DEL -CLARO, K. Influence of extrafloral nectary phenology on antplant mutualistic networks in a neotropical savanna. Ecological Entomology, v. 38, n. 5, p. 463-469. 2009.

MCCLUSKEY, E. S.; BROWN. W. L. Rhythms and other biology of the giant tropical ant Paraponera. Psyche, v. 79, p. 335-347, 1972.

MONDAL, A. K.; CHAKRABORTY, T. MONDAL (PARUI), S. Ant foraging on extrafloral nectaries (EFNs) of Ipomoea pes-caprae (Convolvulaceae) in the dune vegetation: Ants as potential antiherbivore agents. India Journal of Geo-Marine Sciences, v. 42, n. 1, p. 67-74. 2013.

MOREAU, C. S.; BELL, C. D.; VILA, R.; ARCHIBALD, B.; PIERCE, N. E. Phylogeny of the ants: diversification in the age of angiosperms, Science, v. 312, n. 5770, 101-104, 2006.

MOREIRA, V. S. S.; DEL-CLARO, K. The Outcomes of an Ant-Treehopper Association on Solanum lycocarpum St. Hill: Increased Membracid Fecundity and Reduced Damage by Chewing Herbivores. Neotropical Entomology, v. 34, n. 6, p. 881-887, 2005.

MOREIRA, V. S. S.; SEBAIO, F.; DEL-CLARO, K. Desenvolvimento de Enchenopa brasiliensis Strümpel (Homoptera, Membracidae) em plantas de Solanum lycocarpum St. Hill. (Solanaceae) no cerrado e as formigas associadas. Revista brasileira de Zoociências, v. 2, n. 1, p. 21-30, 2000.
MOYA-RAYGOZA, G.; NAULT, L. R. Obligatory Mutualism between Dalbulus quinquenotatus (Homoptera: Cicadellidae) and Attendant Ants. Annals of the Entomological Society of America, v. 93, n. 4, p. 929-940, 2000.

MYERS, J. G. Observations on the biology of two remarkable cixiid plant-hoppers (Homoptera) from Cuba. Psyche, v. 34, p. 283-292, 1929.

NAHAS, L.; DEL-CLARO, K. Ant-plant interactions: absolute frequency as a better method to sample visiting ants in the extrafloral nectary-bearing plant, Lualea multiflora (Vochysiaceae). Sociobiology, v. 57, p. 253-259. 2011.

NASCIMENTO, E. A.; DEL-CLARO, K. Ant visitation to extrafloral nectaries decreases herbivory and increases fruit set in Chamae cristadebilis (Fabaceae) in a Neotropical savanna. Flora, v. 205, p. 754-756, 2010.

NÚÑEZ, J. L. A.; NAYA, M.; CALCAGNOPISSARELLI, M. P.; OTERO, D. Behaviour of Odontomachus chelifer (Latreille) (Formicidae: Ponerinae) feeding on sugary liquids. Journal Insect Behaviour, v. 24, p. 220-229, 2011.

OLIVEIRA, P. S.; FREITAS, A. V. L. Ant-plantherbivore interactions in the Neotropical cerrado savanna. Naturwissenschaften, v. 91, p. 557-570, 2004.

OLIVEIRA, P. S.; PIE. M. R. Interaction between ants and plants bearing extrafloral nectaries in cerrado vegetation. Anais da Sociedade Entomológica do Brasil, v. 27, p. 161-76, 1998.

PASSERA, L.; LACHAUD, J.-P.; GOMEL, L. Individual food source fidelity in the Neotropical ponerine ant Ectatomma ruidum Roger (Hymenoptera Formicidae). Ethology Ecology; Evolution, v. 6, p. 1321, 1994.

PAUL, J.; ROCES, F. Fluid intake rates in ants correlate with their feeding habits. Journal of Insect Physiology, v. 49, p. 347-357, 2003.

PÉREZ-LACHAUD, G.; HERATY, J. M.; CARMICHAEL, A.; LACHAUD, J-P. Biology and behavior of Kapala (Hymenoptera: Eucharitidae) attacking Ectatomma, Gnamptogenys, and Pachycondyla (Formicidae: Ectatomminae and Ponerinae) in Chiapas, Mexico. Annals of Entomological Society of America, v. 99, n. 3, p. 567-576, 2006.

PERFECTO, I.; VANDERMEER, J. The effect of an ant-hemipteran mutualism on the coffee berry borer (Hypothenemus hampei) in southern Mexico. Agriculture, Ecosystems and Environment, v. 117, n. 218-221, 2006. 
PRATT, S. C. 1989. Recruitment and other communication behavior in the ponerinae ant Ectatomma ruidum. Ethology, 81:313-331.

RICKSON, F. R.; RICKSON, M. M. The cashew nut, Anacardium occidentale (Anacardiaceae), and its perennial association with ants: Extrafloral nectary location and the potential for ant defense. American Journal of Botany, v. 85, p. 835-849. 1998.

RICO-GRAY, V.; OLIVEIRA, P. S. The Ecology and Evolution of Ant - Plant Interactions. - Univ. of Chicago Press. 2007.

RODRIGUES, W. C.; CASSINO, P. C. R. Interação entre formigas e aleirodídeos (Sternorrhyncha, Aleyrodidae) em cultivo orgânico de tangerina cv. poncã (Citrus reticulata Blanco). Entomo Brasilis, v. 4, n. 3, p. 119124. 2011.

SANTOS, J. C.; DEL-CLARO, K. Interação entre formigas, herbívoros e nectários extraflorais em Tocoyena formosa (Cham.; Schlechtd.) K. Schum. (Rubiaceae) na vegetação do cerrado. Revista Brasileira de Zoociências, v. 3, n. 1, p. 77-92. 2001.

SCHATZ, B.; LACHAUD, J. P.; BEUGNON, G. Spatial fidelity and individual foraging specializations in the Neotropical ponerine ant, Ectatomma ruidum Roger. Sociobiology, v. 26, p 269-282, 1995.

SCHEMSKE, D. W. The evolutionary significance of extrafloral nectar production by Costus woodsonii (Zingiberaceae): An experimental analysis of ant protection. Journal of Ecology, v. 68, p. 959-67, 1980.

SCHEMSKE, D. W. Ecological correlates of a Neotropical mutualism: ant assembleages at Costus extrafloral nectaries. Ecology, v. 63, p. 932-41, 1982.

SOUZA, T. S.; FRANCINI, R. B. First record of trophobiotic interaction between a ponerine ant and a cicadelid bug. Psyche, v. 2010, Article ID 372385, 4 pgs.

STADLER, B.; DIXON, T. Mutualism: Ants and their insect partners, Cambridge: Cambridge University Press. 2008.
STYRSKY, J. D.; EUBANKS, M. D. Ecological consequences of interactions between ants and honeydew-producing insects. Proceedings of the Royal Society of London. Serie B, v. 274, p. 151-164, 2007.

VALENZUELA-GONZÁLEZ, J.; LÓPEZ-MÉNDEZ A.; LACHAUD J. P. Activity patterns and foraging activity in nests of Ectatomma tuberculatum (Hymenoptera: Formicidae) in cacao plantations. Southwestern Entomologist, v. 20, n. 4, p. 507-515. 1995.

VANDERMEER, J.; PERFECTO, I.; IBARRA NUÑEZ, G.; PHILPOTT, S.; GARCIA BALLINAS, A. Ants (Azteca sp.) as potential biological control agents in shade coffee production in Chiapas, Mexico. Agroforestry Systems. 56, 271-276. 2002.

VILELA, A. A.; TOREZAN-SILINGARDI, H. M.; DEL-CLARO, K. Conditional outcomes in ant-plantherbivore interactions influenced by sequential flowering. Flora, n. 209, p. 359-366. 2014.

WAY, M. J. Mutualism between ants and honeydew producing Homoptera. Annual Review of Entomology, 8:307-344. 1963.

WAY, M. J.; KHOO, K. C. Role of ants in pest management. Annual Review of Entomology, v. 37, 479-503. 1992.

WEBER, N. A. Two common ponerine ants of possible economic significance, Ectatomma tuberculatum (Olivier) and E. ruidum Roger. Proceedings of the Entomological Society of Washington, v. 48: 1-16. 1946.

WILSON E. O.; HÖLLDOBLER. The rise of the ants: A phylogenetic and ecological explanation. Proceedings of the Natural Academy of Science USA, v. 102, n. 21, p. 7411-7414. 2005. 